\section{Designing Output-Feedback Predictive Controllers by Reverse-Engineering Existing LTI Controllers}

\author{
Edward N. Hartley and Jan M. Maciejowski Fellow, IEEE
}

\begin{abstract}
An approach to designing a constrained output-feedback predictive controller that has the same small-signal properties as a preexisting output-feedback linear time invariant controller is proposed. Systematic guidelines are proposed to select an appropriate (non-unique) realisation of the resulting state observer. A method is proposed to transform a class of offset-free reference tracking controllers into the combination of an observer, steady-state target calculator and predictive controller. The procedure is demonstrated with a numerical example.
\end{abstract}

\section{INTRODUCTION}

Handling input and output constraints in a systematic manner is one of the main motivations for the use of model predictive control (MPC) [1]-[3] and is the keystone of its industrial success [4]. The definition of the constraints is usually obvious, corresponding to physical limitations and performance requirements. However, encoding the remaining objectives in the cost function can be difficult.

A linear time-invariant (LTI) baseline controller may already exist for a given application. If full state measurements or estimates are available, and the pre-existing controller is a static state feedback gain, then an inverse optimal cost function may be found. For the unconstrained linear quadratic regulator problem, [5] characterises the set of state feedback gains that are optimal for some quadratic cost function. When a state feedback gain satisfies these conditions, one way of computing corresponding quadratic cost weightings is to pose a linear matrix inequality (LMI) problem [6]. In [7] a quadratic cost function with time-varying weights over a finite prediction horizon is proposed allowing reproduction of a wider range of gains. In [8] a cost function with cross terms between state and input values is shown to reproduce any multivariable state feedback gain. In [7] a method is shown that can reproduce closed-loop behaviour of an output-feedback controller by including the original controller within the plant model, and (non-minimally) parameterising the state of the enlarged system in terms of a finite sequence of previous outputs and inputs. In [9] it is shown that an MPC controller can be constructed to match the unconstrained behaviour of a given $H_{\infty}$ controller, which was designed using the loop-shaping procedure of [10].

In the present work, a method is proposed using the observercompensator realisation of a more general class of stabilising LTI output-feedback controllers (originally proposed by [11], [12], and further developed in [13], [14]), as the basis for a state observer and cost function in an output feedback MPC controller. The methodology in this technical note is motivated by the cross standard form [14] [16], an $\mathcal{H}_{2}$ and $\mathcal{H}_{\infty}$ inverse-optimal generalised plant model whose optimal solutions are the observer-based realisations of a pre-specified output feedback controller $K_{0}$, and builds upon [17]-[20].

The present technical note proposes methods for selecting the (nonunique) observer-based realisation, including the design of additional modes in the observer introduced in the kernel of the initial compensator gain when the plant is of higher order than the baseline controller. The method is extended to transform an LTI offset-free tracking controller into the form of an observer, MPC controller and target calculator [21]-[23].

Manuscript received September 12, 2011; revised June 6, 2012, November 12, 2012, and January 30, 2013. This work was supported by EPSRC grant EP/G030308/1, the European Space Agency and EADS Astrium.

Edward N. Hartley and Jan M. Maciejowski are with the University of Cambridge Department of Engineering (e-mail: \{enh20,jmm\}@eng.cam.ac.uk)
The remainder of this technical note is organised as follows: Section II presents pre-requisites and assumptions; Section III recapitulates the principles for obtaining a discrete-time observer-based realisation of an LTI regulator in sufficient detail to motivate the subsequent design, and proposes a systematic method to help select a non-unique realisation; Section IV transforms the resulting observerbased realisations into an output-feedback MPC regulator; Section V extends the methodology to the case where the baseline controller exhibits integral action for offset-free tracking, by transforming the baseline controller into a target calculator and constrained MPC controller; Section VI presents a numerical example; and Section VII concludes.

\section{PRE-REQUisites AND ASSUMPTIONS}

Assumption 1: The LTI plant to be controlled has a minimal realisation $x(k+1)=A x(k)+B u(k), y(k)=C x(k)$, where $x(k) \in \mathbb{R}^{n_{x}}$ is the plant state, $u(k) \in \mathbb{R}^{n_{u}}$ is the plant input (manipulated variable), $y(k) \in \mathbb{R}^{n_{y}}$ is the plant output (measured variable) at time $k$ and $\operatorname{det}(A) \neq 0$.

Assumption 2: The pair $(C, A)$ is observable, and the tuple $(C, A, B)$ is output stabilisable.

The plant model can consider integrating disturbances, so stabilisability of $(A, B)$ is not assumed.

Assumption 3: The pre-existing output-stabilising LTI regulator for the plant in Assumption 1 has minimal realisation $x_{K}(k+1)=$ $A_{K} x_{K}(k)+B_{K} y(k), u(k)=C_{K} x_{K}(k)+D_{K} y(k)$, where the controller state $x_{K} \in \mathbb{R}^{n_{K}}, n_{K} \leq n_{x}$ and $\operatorname{det}\left(A_{K}\right) \neq 0$.

This excludes controllers including a pure time delay.

Definition 1: For a matrix $T$ with full row rank, let $T^{+}$denote the Moore-Penrose pseudo-inverse of $T$, and $\operatorname{Ker}(T)$ denote the kernel of $T$. Let $T^{\perp}$ be a matrix whose columns form an orthonormal basis for $\operatorname{Ker}(T)$ and $\operatorname{spec}(A)$ denote the set of eigenvalues of $A$.

\section{OBSERVER-BASED CONTROLLER REALISATIONS}

This section summarises the method to obtain an observer-based controller realisation, based on an adaptation of the method for low order controllers from [14] to discrete time. Systematic guidelines for selecting a particular realisation are proposed.

\section{A. Obtaining observer-based controller realisations}

When $D_{K} \neq 0$, a controller based on a filter-form observer is sought, enabling reproduction of the feed-through term from the measured variable to the manipulated variable. Denoting $\hat{x}(k+1 \mid k)$ as the estimate of the state at time instant $k+1$ given measurements at time $k, \hat{x}(k \mid k)$ as the estimate at time $k$ given current measurements, $D_{Q}$ as a static Youla parameter [24], and $A_{f}=\left(A-A K_{f} C\right)$ for $K_{f} \in \mathbb{R}^{n_{x} \times n_{y}}$, this is

$$
\begin{aligned}
\hat{x}(k+1 \mid k) & =A_{f} \hat{x}(k \mid k-1)+B u(k)+A K_{f} y(k) \\
\hat{x}(k \mid k) & =\left(I-K_{f} C\right) \hat{x}(k \mid k-1)+K_{f} y(k) \\
y_{Q} & =D_{Q}(y(k)-C \hat{x}(k \mid k-1)) \\
u(k) & =K_{c} \hat{x}(k \mid k)+y_{Q}(k) .
\end{aligned}
$$

Theorem 1 (Proof in appendix): Given a stabilising regulator satifying Assumption 3, assume that there exists $T \in \mathbb{R}^{n_{K} \times n_{x}}$ of full row rank satisfying the non-symmetric Riccati equation

$$
-T\left(A+B D_{K} C\right)-T B C_{K} T+B_{K} C+A_{K} T=0 .
$$

The regulator can be realised in the form (1), where $A K_{f}=$ $T^{+} B_{K}-B D_{K}+T^{\perp} K_{N}, K_{c}=D_{K} C+C_{K} T, D_{Q}=\left(D_{K}-\right.$ $\left.K_{c} K_{f}\right)$, and $K_{N} \in \mathbb{R}^{\left(n_{x}-n_{K}\right) \times n_{y}}$ is a design parameter. 
Corollary 1 (Proof in appendix): When $A_{K}$ is invertible, and $D_{K}=C_{K} A_{K}^{-1} B_{K}$, then $K_{c} K_{f}=D_{K},[11]$ and therefore $y_{Q}=0$. If $D_{K}=0$, a predictor form observer based controller is sought where (defining $A_{f p}=A-K_{f} C$ ):

$$
\begin{aligned}
\hat{x}(k+1 \mid k) & =A_{f p} \hat{x}(k \mid k-1)+B u(k)+K_{f} y(k) \\
u(k) & =K_{c} \hat{x}(k \mid k-1) .
\end{aligned}
$$

Analogous to Theorem 1, (3) is equivalent to a (possibly nonminimal) realisation of the given controller, with $K_{f}=T^{+} B_{K}+$ $T^{\perp} K_{N}$ and $K_{c}=C_{K} T$, where $-T A-T B C_{K} T+B_{K} C+A_{K} T=$ 0 . If a predictor form is preferred, $D_{K} \neq 0$, and inserting a delay to force $D_{K}=0$ is not acceptable, the transformation $A \leftarrow A+B D_{K} C$ and $D_{K} \leftarrow 0$ can be used, but if $u(k)=K_{c} \hat{x}(k \mid k-1)$ is replaced by a constrained controller, then a disturbance can cause an input constraint violation, since correction occurs at the next time step.

\section{B. Selection of non-unique realisation}

A realisation obtained from application of Theorem 1 (or equivalent for the predictor) is not in general unique. The specific $T$ satisfying (2) determines the partition of the original closed loop eigenvalues between $\operatorname{spec}\left(A+B K_{c}\right)$ and $\operatorname{spec}\left(A-A K_{f} C\right)$ (or $\operatorname{spec}\left(A-K_{f} C\right)$ for a predictor). See [11]-[14], [17], [18] for details.

Even if process and measurement noise are additive, Gaussian and white, with respective covariances $Q_{y}$ and $R_{y}, K_{f}$ designed as described in Section III-A is not (in general) the optimal Kalman gain for the plant for the given covariances. However, estimation error covariance can still be minimised within the degrees of freedom in $T$. For the case when $n_{x}=n_{K}$, this is summarised in Algorithm 1 .

Algorithm 1: $\min _{T, K_{f}, P_{y}} \operatorname{Tr}\left(\left(I-K_{f} C\right) P_{y}\left(I-K_{f} C\right)^{T}+\right.$ $\left.K_{f} R_{y} K_{f}^{T}\right)$ subject to $A_{f} P_{y} A_{f}^{T}-P_{y}=-A K_{f} R_{y} K_{f}^{T} A^{T}-Q_{y}$, $T\left(A+B D_{K} C\right)+T B C_{K} T=B_{K} C+A_{K} T$, and $A K_{f}=$ $T^{+} B_{K}-B D_{K}$.

This can be computed by enumeration of each valid $T$ (see e.g. [13] for restrictions). When $n_{K}<n_{x}, n_{x}-n_{K}$ extra eigenvalues are introduced into $\operatorname{spec}\left(A-A K_{f} C\right)$, corresponding to eigenvectors in $\operatorname{Ker}(T)$ as revealed by similarity transformation on $\left(A-A K_{f} C\right)$. Defining $\tilde{A}=A+B D_{K} C$,

$$
\begin{aligned}
& {\left[\begin{array}{c}
T \\
T^{\perp^{T}}
\end{array}\right]\left[\tilde{A}-T^{+} B_{K} C-T^{\perp} K_{N} C\right]\left[\begin{array}{cc}
T^{+} & T^{\perp}
\end{array}\right]} \\
& \quad=\left[\begin{array}{cc}
T \tilde{A} T^{+}-B_{K} C T^{+} & 0 \\
T^{\perp^{T}} \tilde{A} T^{+}-K_{N} C T^{+} & T^{\perp^{T}} \tilde{A} T^{\perp}-K_{N} C T^{\perp}
\end{array}\right]
\end{aligned}
$$

Determined by $K_{N}$, these do not change the unconstrained, closedloop behaviour with $u(k)=K_{c} \hat{x}(k)$ (one can verify that $\left(K_{c}(I-\right.$ $\left.\left.K_{f} C\right)-D_{Q} C\right) T^{\perp}=0$ and therefore that $T^{\perp^{T}} \hat{x}(k \mid k-1)$ does not contribute to control action (1) (see Appendix)), but do affect the complete state estimate and therefore the closed-loop behaviour with constrained predictive control.

Noting that the estimate of $T x(k)$ is in general not optimal for the given $Q_{y}$ and $R_{y}$, the corresponding estimation error cannot be assumed to be Gaussian white noise, precluding de-coupled design of the estimate of $T^{\perp} x(k)$. For a given $T$ satisfying (2), and defining $A K_{f 0}=T^{+} B_{K}-B D_{K}, A K_{f}=A K_{f 0}+T^{\perp} K_{N}$ and $A_{f}=A-A K_{f} C$, minimising the trace of $P_{0}$ over stabilising $K_{N}$ subject to $P_{0}=A_{f} P_{0} A_{f}^{T}+\left(A K_{f 0}+T^{\perp} K_{N}\right) R\left(A K_{f 0}+T^{\perp} K_{N}\right)^{T}$ corresponds to a dual of the static output feedback discrete-time linear quadratic control problem, which is known to be challenging [25]. Whilst algorithms exist that are often capable of identifying adequate suboptimal solutions (e.g. [26]-[28]), convergence is not guaranteed. Their use in finding a solution $A K_{f}=T^{\perp} B_{K}+D_{K} C+T^{\perp} K_{N}$ in the context of Algorithm 1 is limited if there is more than a handful of candidate matrices $T$ to evaluate, due to computational demands.

When the set of matrices $T$ to evaluate is larger, an optimistic approximation of the achievable estimation error may be used. Define $\hat{x}_{T}(k \mid k-1) \in \mathbb{R}^{n_{K}}$ as an estimate of $T x, \hat{x}_{1}(k \mid k-1) \in \mathbb{R}^{n_{x}}$ as a second estimate of the full state, and $L$ as the Kalman gain for the given uncertainty covariances $Q_{y}$ and $R_{y}$, and defining $A_{L}=$ $(A-A L C)$ :

$$
\begin{aligned}
& \hat{x}_{T}(k \mid k-1)=T\left(A_{f} T^{+} \hat{x}_{T}(k)+B u(k)+A K_{f} y(k)\right) \\
& \hat{x}_{1}(k \mid k-1)=A_{L} \hat{x}_{1}(k \mid k-1)+B u(k)+A L y(k) \\
& \hat{x}(k \mid k)=K_{c}^{+} K_{c}\left(I-K_{f} C\right) T^{+} \hat{x}_{T}(k \mid k-1) \\
&+K_{c}^{\perp} K_{c}^{\perp^{T}}(I-L C) \hat{x}_{1}(k \mid k-1)+D_{\text {obs }} y(k) \\
& D_{\text {obs }}=K_{c}^{+} K_{c} K_{f}+K_{c}^{\perp} K_{c}^{\perp^{T}} L \\
& u(k)=K_{c} \hat{x}(k \mid k)+D_{Q} T^{+} \hat{x}_{T}\left(y(k)-C T^{+} \hat{x}_{T}(k \mid k-1)\right) .
\end{aligned}
$$

This structure combines the reverse-engineered observer of the component of $x(k)$ in $\operatorname{Span}\left(K_{c}\right)$ with a separate estimate of $x(k)$ in $\operatorname{Ker}\left(K_{c}\right)$. The covariance of the error of estimate (5c) is an approximation from below of that which could be obtained using structure (1) with $K_{c}$ and $A K_{f}$ from Theorem 1, and enables a computationally tractable estimate that can be used within the framework of a modified Algorithm 1 when $n_{K}<n_{x}$. For $T$ yielding the lowest bound, $K_{N}$ which approximately minimises $\operatorname{Tr}\left(P_{0}\right)$ within the structure (1) with gains from Theorem 1, can be found using (e.g.) one of the algorithms of [26]-[28] (noting the cross covariance between measurement and process uncertainty, induced by $A K_{f 0}$ ).

Remark 1: An alternative goal is to use $K_{N}$ to force $D_{Q} \rightarrow 0$. Each of the $n_{x}-n_{K}$ modes of (1) in $\operatorname{Ker}(T)$ is cancelled by a corresponding invariant zero. If the plant model is augmented with a disturbance on each channel (subject to observability), placing the resulting eigenvalues in the observer error dynamics at the origin introduces corresponding invariant zeros. However, in Section V, the Youla parameter and disturbance states are treated in the same way.

\section{INVERSE OPTIMAL PREDICTIVE REGULATOR}

This section assumes a perfect plant-model match, that the observer error has converged to zero, that the Youla parameter $D_{Q}=0$ (relaxed subsequently) and that exact matching of $K_{c}$ is required in some neighbourhood of the origin. Define $u_{i} \in \mathbb{R}^{n_{u}}$ as a predicted input $i$ time steps in the future, and $x_{i} \in \mathbb{R}^{n_{x}}$ as a predicted future state, let $\mathbf{u}=\left[u_{0}, \ldots, u_{N-1}\right]^{T}$ for a finite prediction horizon $N \in \mathbb{N}^{+}$and let $\hat{x}(k)$ be the current relevant state estimate $\hat{x}(k \mid k)$ or $\hat{x}(k \mid k-1)$, in this case from (1b), (3a) or (5c) with gains obtained by application of Algorithm 1 and Theorem 1 (or the predictor-form equivalent).

Algorithm 2 (Linear model predictive control): At each time step, $k$, let $\mathbf{u}^{*}=\arg \min _{\mathbf{u}} F_{N}\left(x_{N}\right)+\sum_{i=0}^{N-1} \ell\left(x_{i}, u_{i}\right)$ subject to $x_{0}=$ $\hat{x}(k), x_{i+1}=A x_{i}+B u_{i}, u_{i} \in \mathbb{U}, x_{i} \in \mathbb{X}$, for $i \in\{0, \ldots, N-1\}$ and $x_{N} \in \mathbb{T}$. Apply $u(k)=u_{0}^{*}$. Increment $k$.

Sets $\mathbb{U} \subseteq \mathbb{R}^{n_{u}}, \mathbb{X} \subseteq \mathbb{R}^{n_{x}}$ and $\mathbb{T} \subseteq \mathbb{R}^{n_{x}}$ are assumed to be convex, compact, polytopic, and to contain the origin within their interiors. $\mathbb{T}$ is assumed to be a positively invariant admissible set under the action of the controller $u(k)=K_{c} x(k): \mathbb{T} \triangleq$ $\left\{x_{N}: x_{N} \in \mathbb{T} \Longrightarrow x_{N} \in \mathbb{X}, K_{c} x_{N} \in \mathbb{U},\left(A+B K_{c}\right) x_{N} \in \mathbb{T}\right\}$.

Letting the function $c(\cdot)$ be a continuous, strictly increasing function with $c(0)=0$, [29] shows that for appropriate $\ell\left(x_{i}, u_{i}\right)>c\left(\left\|\left(x_{i}, u_{i}\right)\right\|\right)^{2}$, the value function can be used as a control Lyapunov function to prove stability of the closed loop system with the predictive controller by ensuring $F_{N}\left(A x_{N}+B K_{c} x_{N}\right)-F_{N}\left(x_{N}\right) \leq-\ell\left(x_{N}, K_{c} x_{N}\right)$. 
The reverse engineering procedure hinges upon replacing $K_{c}$ as defined in Theorem 1 with a constrained predictive regulator that is identically equivalent when constraints are not active. For $R>0 \in$ $\mathbb{R}^{n_{u} \times n_{u}}$, the stage cost

$$
\begin{aligned}
\ell_{0}\left(x_{i}, u_{i}\right) & =\left(u_{i}-K_{c} x_{i}\right)^{T} R\left(u_{i}-K_{c} x_{i}\right) \\
& =\left[\begin{array}{l}
x_{i} \\
u_{i}
\end{array}\right]^{T}\left[\begin{array}{cc}
K_{c}^{T} R K_{c} & -K_{c}^{T} R \\
-R K_{c} & R
\end{array}\right]\left[\begin{array}{l}
x_{i} \\
u_{i}
\end{array}\right]
\end{aligned}
$$

where $K_{c}$ is the gain matrix obtained from Theorem 1 gives an unconstrained optimum $u_{i}=K_{c} x_{i}$ [8] with infinite horizon costto-go, $F_{N}(x)=0$, (verifiable by direct substitution into discretetime algebraic Riccati equation with cross-terms). $\ell_{0}\left(x_{i}, u_{i}\right)=0$ if $u_{i}=K_{c} x_{i}$; however, $\ell_{0}\left(x_{i}, u_{i}\right)$ is not bounded below by $c\left(\left\|x_{i}, u_{i}\right\|\right)^{2}$. To circumvent this, a dual-mode approach with cost

$$
\ell\left(x_{i}, u_{i}\right)=\ell_{0}\left(x_{i}, u_{i}\right)+\left(x_{i}-z_{i}\right)^{T} Q_{1}\left(x_{i}-z_{i}\right)
$$

subject to slack variable $z_{i} \in \alpha \mathbb{T} \subset \mathbb{R}^{n_{x}}$, for design parameters $Q_{1}>0 \in \mathbb{R}^{n_{x} \times n_{x}}$ and $0 \leq \alpha<1$ can be shown to guarantee finite-time entry to $\mathbb{T}$, wherein the unconstrained (known stabilising) control gain is recursively feasible [30].

An alternative cost, without recourse to slack variables, of the form

$$
\ell_{S}\left(x_{i}, u_{i}\right)=x_{i}^{T} Q x_{i}+x_{i}^{T} S u_{i}+u_{i} S^{T} x_{i}+u_{i}^{T} R u_{i}
$$

can be used, accompanied by terminal cost $F_{N}\left(x_{N}\right)=x_{N}^{T} P x_{N}$. If $(A, B)$ is stabilisable, and there exist appropriately dimensioned $Q>$ $0, R>\epsilon I, S, P>\delta I$ (for scalar bounds $\epsilon>0$ and $\delta>0$ ), satisfying $Q-S R^{-1} S^{T}>0, A^{T} P A-P-K_{c}^{T}\left(B^{T} P B+R\right) K_{c}+Q=0$, $\left(B^{T} P B+R\right) K_{c}+\left(B^{T} P A+S^{T}\right)=0$ and, letting $A_{c}=\left(A+B K_{c}\right)$,

$$
A_{c}^{T} P A_{c}-P+Q+K_{c}^{T} R K_{c}+K_{c}^{T} S^{T}+S K_{c} \leq 0
$$

then the matrices $Q, R, S$ and $P$ in cost function (8) will be inverse optimal with respect to the static gain $K_{c}$, and the terminal cost $F_{N}\left(x_{N}\right)$ will be a control Lyapunov function within $\mathbb{T}$, enabling constrained closed-loop stability to be established using the method of [29]. This can be solved using convex methods [31], with any additional degrees of freedom used to minimise $\|S\|_{2}^{2}$.

In principle, the effect of the Youla parameter, when $D_{Q} \neq 0$ can be considered by substituting $\ell\left(x_{i}, u_{i}\right)$, with $\ell\left(x_{i}, u_{i}-y_{Q}(k)\right)$ in Algorithm 2. However, whilst $y_{Q}(k) \rightarrow 0$ as $k \rightarrow \infty$, a given pair $\left(0, y_{Q}(k)\right)$ is not necessarily an equilibrium pair, and it might therefore be preferable to operate within the same framework as for offset-free tracking, as elaborated subsequently.

\section{REVERSE-ENGINEERED REFERENCE TRACKING CONTROLLERS}

This section extends the proposed approach with the treatment of a class of offset-free reference tracking controllers. The subsequent analysis assumes a filter form observer, but analogous results hold for the predictor form.

Direct application of Theorem 1 and cost function (6) would reproduce the unconstrained controller, but the observer can be biased by disturbances, and thus prediction quality, and consequently closed loop performance in presence of constraints, can be compromised. The following development transforms a baseline controller into a widely used form for offset-free predictive control [21]-[23].

\section{A. Further standing assumptions and pre-requisites}

Assumption 4: The baseline, stabilising, linear, time-invariant, offset-free, reference tracking controller has a minimal realisation

$$
\begin{aligned}
x_{K}(k+1) & =A_{K} x_{K}(k)+B_{K}(y(k)-r(k)) \\
u(k) & =C_{K} x_{K}(k)+D_{K}(y(k)-r(k)),
\end{aligned}
$$

where the controller state $x_{K} \in \mathbb{R}^{n_{K}}$ and $r(k) \in \mathbb{R}^{n_{y}}$ is a reference which tends to a constant $r_{\infty}$ as $k \rightarrow \infty$. $A_{K}$ has at least $n_{y}$ eigenvalues at 1 , with a corresponding invariant subspace orthogonal to neither the rows of $C_{K}$, nor the columns of $B_{K}$. In closed loop with this controller, the plant output $y(k)$ tends to $r(k)$ as $k \rightarrow \infty$.

Assumption 5: The pair $(A, B)$ is stabilisable, and a matrix $B_{d}$ has been chosen so that the plant model can be augmented as

$$
\begin{aligned}
{\left[\begin{array}{c}
x(k+1) \\
d(k+1)
\end{array}\right] } & =\underbrace{\left[\begin{array}{cc}
A & B_{d} \\
0 & I
\end{array}\right]}_{A_{a}} \underbrace{\left[\begin{array}{c}
x(k) \\
d(k)
\end{array}\right]}_{x_{a}(k)}+\underbrace{\left[\begin{array}{c}
B \\
0
\end{array}\right]}_{B_{a}} u(k) \\
y(k) & =\underbrace{\left[\begin{array}{ll}
C & 0
\end{array}\right]}_{C_{a}}\left[\begin{array}{l}
x(k) \\
d(k)
\end{array}\right]
\end{aligned}
$$

where $d(k) \in \mathbb{R}^{n_{y}}$ is an uncontrollable disturbance state, the pair $\left(C_{a}, A_{a}\right)$ is detectable. The input dimension $n_{u} \geq n_{y}$.

By applying Theorem 1 to plant (11) and controller (10), and separating the contributions of $r(k)$ and $y(k)$ to $u(k)$, a controller realisation with the structure of an observer estimating $x_{a}(k)$ and a reference pre-filter with internal state $\hat{x}_{P, a}(k)=\left[\hat{x}_{P}^{T}(k), \hat{d}_{P}^{T}(k)\right]^{T}$ is obtained (letting $A_{a f}=\left(A_{a}-A_{a} K_{f} C_{a}\right)$ ):

$$
\begin{aligned}
\hat{x}_{a}(k+1 \mid k) & =A_{a f} \hat{x}_{a}(k \mid k-1)+B_{a} u(k)+A_{a} K_{f} y(k) \\
\hat{x}_{a}(k \mid k) & =\left(I-K_{f} C\right) \hat{x}_{a}(k \mid k-1)+K_{f} y(k) \\
y_{Q}(k) & =D_{Q}\left(y(k)-C \hat{x}_{a}(k \mid k-1)\right) \\
\hat{x}_{P, a}(k+1 \mid k) & =A_{a f} \hat{x}_{P, a}(k \mid k-1)+A_{a} K_{f} r(k) \\
\hat{x}_{P, a}(k \mid k) & =\left(I-K_{f} C\right) \hat{x}_{P, a}(k \mid k-1)+K_{f} r(k) \\
q_{P}(k) & =D_{Q}\left(r(k)-C \hat{x}_{P, a}(k \mid k-1)\right) \\
u(k) & =K_{c}\left(\hat{x}_{a}(k \mid k)-\hat{x}_{P, a}(k \mid k)\right)+y_{Q}(k)-q_{P}(k) .
\end{aligned}
$$

\section{B. Target equilibrium pairs}

Partition $K_{f}$ and $K_{c}$ as $K_{f}=\left[\begin{array}{ll}K_{f 1}^{T} & K_{f d}^{T}\end{array}\right]^{T}$ and $K_{c}=$ $\left[\begin{array}{ll}K_{c 1} & K_{c d}\end{array}\right]$ where $K_{f 1} \in \mathbb{R}^{n_{x} \times n_{y}}, K_{f d} \in \mathbb{R}^{n_{y} \times n_{y}}, K_{c 1} \in$ $\mathbb{R}^{n_{u} \times n_{x}}$ and $K_{c d} \in \mathbb{R}^{n_{u} \times n_{y}}$.

The pair $\left(x_{s}(k), u_{s}(k)\right)$ is an equilibrium pair, satisfying $C x_{s}=$ $C \hat{x}_{P}$ and $u(k)=K_{c 1}\left(\hat{x}(k \mid k)-x_{s}(k)\right)+u_{s}(k)$ is equal to that of the baseline controller, if

$$
\begin{gathered}
{\left[\begin{array}{cc}
(A-I) & B \\
C & 0 \\
-K_{c 1} & I
\end{array}\right]\left[\begin{array}{l}
x_{s}(k) \\
u_{s}(k)
\end{array}\right]=G\left[\begin{array}{lllll}
\hat{d}^{T} & y_{Q}^{T} & \hat{d}_{P}^{T} & \hat{x}_{P}^{T} & q_{P}^{T}
\end{array}\right]^{T}(k \mid k)} \\
\text { where } \\
G=\left[\begin{array}{ccccc}
-B_{d} & 0 & 0 & 0 & 0 \\
0 & 0 & C & 0 & 0 \\
K_{c d} & I & -K_{c d} & -K_{c 1} & -I
\end{array}\right]
\end{gathered}
$$

The final row is deduced by comparing expressions for the corresponding control actions and cancelling common terms.

Proposition 1: If there exists a vector $x_{\text {ref }}(k) \in \mathbb{R}^{n_{x}}$ such that

$$
K_{c 1} x_{\mathrm{ref}}(k)=K_{c 1} \hat{x}_{P}(k)+K_{c d} \hat{d}_{P}(k)+q_{P}(k)
$$

and there exist $\delta_{1} \in \mathbb{R}^{n_{x}}$ and $\delta_{2} \in \mathbb{R}^{n_{u}}$, such that

$$
\left[\begin{array}{cc}
(A-I) & B \\
C & 0
\end{array}\right]\left[\begin{array}{l}
\delta_{1} \\
\delta_{2}
\end{array}\right]=\left[\begin{array}{l}
0 \\
C
\end{array}\right] x_{\mathrm{ref}}
$$

and that

$$
\left[\begin{array}{ll}
-K_{c 1} & I
\end{array}\right]\left[\begin{array}{l}
\delta_{1} \\
\delta_{2}
\end{array}\right]=-K_{c 1} x_{\mathrm{ref}}+y_{Q}(k),
$$

Preprint submitted to IEEE Transactions on Automatic Control. Received: April 8, 2013 09:54:26 PST 
then the pair $\left(x_{s}(k), u_{s}(k)\right)$ is an equilibrium pair, satisfying $C x_{s}=$ $C \hat{x}_{\text {ref }}$ and the control action $u(k)=K_{c 1}\left(\hat{x}(k \mid k)-x_{s}(k)\right)+u_{s}(k)$ is identical to that of the baseline controller, if

$$
\left[\begin{array}{cc}
(A-I) & B \\
C & 0 \\
-K_{c 1} & I
\end{array}\right]\left[\begin{array}{l}
x_{s}(k) \\
u_{s}(k)
\end{array}\right]=\left[\begin{array}{cc}
-B_{d} & 0 \\
0 & C \\
K_{c d} & -K_{c 1}
\end{array}\right]\left[\begin{array}{c}
\hat{d}(k \mid k) \\
x_{\mathrm{ref}}(k)
\end{array}\right] .
$$

Lemma 1: A sufficient condition for the existence of $x_{\text {ref }}$ satisfying the conditions in Proposition 1 is that $\operatorname{rank}(M) \leq n_{x}-n_{u}$, where

$$
M=\left(\left[\begin{array}{ll}
-K_{c 1} & I
\end{array}\right]\left[\begin{array}{cc}
(A-I) & B \\
C & 0
\end{array}\right]^{+}\left[\begin{array}{l}
0 \\
C
\end{array}\right]+K_{c 1}\right) .
$$

Proof: Noting that $n_{y} \leq n_{u}$, a sufficient condition for satisfaction of the conditions (14b) and (14c) can be obtained by solving (14b) for $\left(\delta_{1}, \delta_{2}\right)$ and substituting into (14c) to obtain the condition $M x_{\text {ref }}=y_{Q}$. However, (14a) must also be required to hold. A sufficient condition for existence is obtained by consideration of the dimensions of the left side of:

$$
\left[\begin{array}{c}
M \\
K_{c 1}
\end{array}\right] x_{\mathrm{ref}}(k)=\left[\begin{array}{cc}
0 & 0 \\
K_{c 1} & K_{c d}
\end{array}\right]\left[\begin{array}{c}
\hat{x}_{P}(k \mid k) \\
\hat{d}_{P}(k \mid k)
\end{array}\right]+\left[\begin{array}{c}
y_{Q}(k) \\
q_{P}(k)
\end{array}\right] .
$$

Lemma 2: When $K_{c 1}$ and $K_{c d}$ are chosen as prescribed, a solution to (15) exists.

Proof: When $C x_{\text {ref }}=0$, Assumption 4 implies that for any $d_{\infty} \in \mathbb{R}^{n_{y}}$, an equilibrium pair $\left(x_{\infty}, u_{\infty}\right)$ must exist, such that $(A-I) x_{\infty}+B u_{\infty}=-B_{d} d_{\infty}$ and $C x_{\infty}=0$. The observerbased realisation of the same controller, with zero reference, gives $u_{\infty}=K_{c 1} x_{\infty}+K_{c d} d_{\infty}$. For $C x_{\text {ref }} \neq 0$, choosing $x_{\text {ref }}$ to satisfy (17) implies that there exists $\left(\delta_{1}, \delta_{2}\right)$ satisfying (14b) and (14c). By linear superposition, the pair $\left(x_{s}, u_{s}\right)=\left(x_{s 0}+\delta_{1}, u_{s 0}+\delta_{2}\right)$ satisfies the required conditions.

Remark 2: When $\left[\begin{array}{cc}(A-I) & B \\ C & 0\end{array}\right]$ has a unique inverse, (15) is equivalent to the standard conditions for equilibrium target calculation [21], [22] with reference setpoint $C x_{\text {ref. }}$ Otherwise, the additional constraints use the degrees of freedom to match the input from the original controller. When $D_{Q}=0, x_{\text {ref }}$ is a function only of the pre-filter reference state.

Remark 3: Assumption (4) states that $C x(k) \rightarrow r(k)$ as $k \rightarrow \infty$ for the system in closed loop with (10). Controller (12) is equivalent to (10). Controller $u(k)=K_{c 1}\left(x(k)-x_{s}(k)\right)+u_{s}(k)$, where $\left(x_{s}(k), u_{s}(k)\right)$ satisfy (15) with $x_{\text {ref }}$ satisfying (17), is equivalent to (12). Condition (15) implies that with the aforementioned controller $C x(k) \rightarrow C x_{\mathrm{ref}}(k)$ [21]-[23]. Therefore $C x_{\mathrm{ref}}(k) \rightarrow r(k)$ as $k \rightarrow \infty$.

Remark 4: The objective is only to match the original controller when constraints are not active, so to avoid infeasibility in the presence of plant input and state constraints, similarly to the constraint that $C x_{s}=C x_{\text {ref }}$, it is preferable that these additional conditions $-K_{c 1} x_{s}+u_{s}=K_{c d} \hat{d}-K_{c 1} x_{\text {ref }}$ be implemented as "soft" constraints, that hold when state and input constraints are not active but may be violated in favour of state and input constraints.

\section{Realisation as a predictive controller}

The target equilibrium pair $\left(x_{s}(k), u_{s}(k)\right)$, satisfying (15), where $x_{\text {ref }}(k)$ satisfies $(17)$, and $\hat{x}_{P}(k \mid k), \hat{d}_{P}(k \mid k), y_{Q}(k)$ and $q_{P}(k)$ are obtained by implementing pre-filter and observer (12a-f) is used as a reference setpoint in the predictive controller with the cost function of the form (6), (7) or (8) designed to be inverse-optimal for gain $K_{c 1}$ and plant matrices $(A, B)$.
TABLE I

ALLOCATION OF CLOSED-LOOP DYNAMICS

\begin{tabular}{cccccc}
\hline \hline Pole & 1 & 0.976 & $0.992+j 0.119$ & $0.992-j 0.119$ & 0.558 \\
\hline Opt. 1 & $\mathrm{S}$ & $\mathrm{S}$ & $\mathrm{O}$ & $\mathrm{O}$ & $\mathrm{S}$ \\
Opt. 2 & $\mathrm{S}$ & $\mathrm{O}$ & $\mathrm{S}$ & $\mathrm{S}$ & $\mathrm{O}$ \\
\hline
\end{tabular}

Algorithm 3 (Linear model predictive control with reference tracking): At each time step, $k$, let $\mathbf{u}^{*}=\arg \min _{\mathbf{u}} F_{N}\left(x_{N}-\right.$ $\left.x_{s}\right)+\sum_{i=0}^{N-1} \ell\left(x_{i}-x_{s}, u_{i}-u_{s}\right)$ subject to $x_{0}=\hat{x}(k)$, $x_{i+1}=A x_{i}+B u_{i}+B_{d} \hat{d}(k \mid k), u_{i} \in \mathbb{U}$, for $i \in\{0, \ldots, N-1\}$ and $x_{i} \in \mathbb{X}$, for $i \in\{0, \ldots, N\}$. Apply $u(k)=u_{0}^{*}$. Increment $k$.

\section{NUMERICAL EXAMPLE}

The baseline plant and controller for this numerical example are taken from [32, ex 9.4.1] for a single axis attitude control system with angle measurement (in radians) only, and the applied torque (in $\mathrm{Nm})$ as the plant input. For a sample time $T_{s}=0.25 \mathrm{~s}$, the plant matrices are $A=\left[\begin{array}{cc}1 & 0.25 \\ 0 & 1\end{array}\right], B=\frac{1}{500}\left[\begin{array}{c}0.03125 \\ 0.25\end{array}\right], C=\left[\begin{array}{ll}1 & 0\end{array}\right]$, $D=0$. The controller matrices are $A_{K}=\left[\begin{array}{cc}1.4118 & -0.8235 \\ 0.5 & 0\end{array}\right]$, $B_{K}=\left[\begin{array}{ll}32 & 0\end{array}\right]^{T}, C_{K}=\left[\begin{array}{ll}-13.0133 & -26.1415\end{array}\right]$, and $D_{K}=$ -871.0478 . The poles of the baseline controller are at 1 and 0.4118 , and the poles of the resulting closed-loop system are at 0.5579 , $0.9115 \pm j 0.1192$ and 0.9764 . The augmented system (11) is formed with $B_{d}=B$ (i.e. a torque disturbance). The resulting closed loop system has an additional observable, but uncontrollable pole at $z=1$. Since $D_{K} \neq 0,\left|D_{K}\right| \gg 0$, and $D_{K} \neq C_{K} A_{K}^{-1} B_{K}$, the filter-form observer (1) with static Youla parameter is chosen.

The integrating disturbance is uncontrollable, so must remain in $\operatorname{spec}\left(A_{a}+B_{a} K_{c}\right)$, and the complex conjugate pair cannot be split, resulting in two feasible possible allocations of the closed-loop dynamics between $\operatorname{spec}\left(A_{a}+B_{a} K_{c}\right)$ and $\operatorname{spec}\left(A_{a}-A_{a} K_{f} C_{a}\right)$. For this example, $n_{x}=3$ (due to the disturbance augmentation) but $n_{K}=2$. Therefore, the process will introduce a single additional mode in the observer in the nullspace of $T$. For this example we will assume that $Q_{y}=\operatorname{diag}\left(10^{-6}, 10^{-7}, 10^{-3}\right)$ and $R_{y}=1.2 \times 10^{-7}$. The latter corresponds to an RMS sensor error of $\approx 0.02^{\circ}$.

For each of the options in Table I the observer performance is evaluated in response to process and measurement noise $Q_{y}$ and $R_{y}$ in closed loop with the unconstrained inverse-optimal MPC using cost function (IV) regulating to the origin. The results are shown in Table II. Reproduction of the baseline control input is also verified. The maximum absolute error between the control input from the baseline realisation and the observer-based realisation is denoted $u_{\mathrm{err}}$, and the RMS control inputs applied are presented to verify that the error is negligible in comparison, and denoted $u_{\text {rms }}$. Errors $e_{1}, e_{2}$ and $e_{3}$ denote the RMS error in simulation on states 1,2 and 3 respectively. We denote case (a) where structure (1) with gains chosen using Theorem 1 with $K_{N}$ used to place $\operatorname{spec}\left(T^{\perp^{T}}\left(A-A K_{f} C\right) T^{\perp}\right)=0$ is used as the basis for the design (for this model, this also causes $D_{Q}=0$ ); case (b) where structure (5e) with an augmented observer is used; and case (c) where the degree of freedom $K_{N}$ is used to approximately minimise the estimation error by application of [26]. The LMI solver SeDuMi [33] is used through the YALMIP interface [34] to solve for $K_{N}$ and to find $(Q, R, S, P)$ by solving (IV).

To demonstrate the inheritance of the offset-free tracking and constraint handling, realisation $1(\mathrm{c})$ is chosen (by virtue of Algorithm 1) with gains $\left[K_{c 1}, K_{c d}\right]=[-83.4,-921,-1]$, 
This is the author's version of an article that has been published in this journal. Changes were made to this version by the publisher prior to publication.

The final version of record is available at http://dx.doi.org/10.1109/TAC.2013.2258781

Limited circulation. For review only
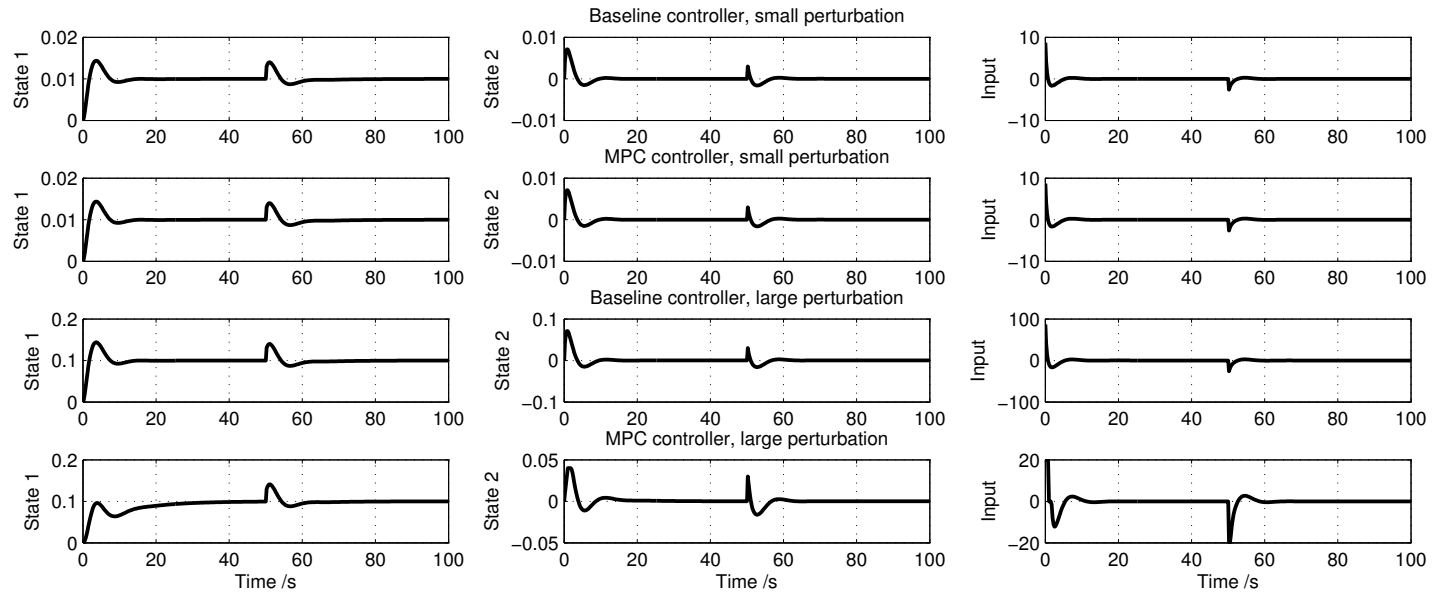

Fig. 1. Closed loop responses to step reference change and input disturbance for baseline and MPC realisation with constraints (note differences in axes)

TABLE II

EVALUATION OF OBSERVER PERFORMANCE

\begin{tabular}{cccc}
\hline \hline Option & $1(\mathrm{a})$ & $1(\mathrm{~b})$ & $1(\mathrm{c})$ \\
\hline $\operatorname{Tr}\left(\hat{P}_{y}\right)^{1 / 2}$ & 0.3865 & 0.17526 & 0.1830 \\
$D_{Q}$ & 0 & -777.0 & -742.7 \\
$e_{1}$ & 0.0004 & 0.0005 & 0.0016 \\
$e_{2}$ & 0.0017 & 0.0040 & 0.0016 \\
$e_{3}$ & 0.3659 & 0.1827 & 0.1859 \\
$u_{\mathrm{rms}}$ & 4.4613 & 4.4613 & 4.4613 \\
$u_{\mathrm{err}}$ & $4 \times 10^{-7}$ & $5 \times 10^{-7}$ & $2 \times 10^{-6}$ \\
\hline \hline & & \\
\hline \hline Option & $2(\mathrm{a})$ & $2(\mathrm{~b})$ & $2(\mathrm{c})$ \\
\hline $\operatorname{Tr}\left(\hat{P}_{y}\right)^{1 / 2}$ & 0.1970 & 0.17539 & 0.1857 \\
$D_{Q}$ & 0 & -777.0 & -656.9 \\
$e_{1}$ & 0.0004 & 0.0097 & 0.0006 \\
$e_{2}$ & 0.0024 & 0.0179 & 0.0016 \\
$e_{3}$ & 0.2127 & 0.1827 & 0.2108 \\
$u_{\max }$ & 4.4613 & 4.4613 & 4.4613 \\
$u_{\mathrm{err}}$ & $9 \times 10^{-4}$ & $2 \times 10^{-3}$ & $1 \times 10^{-3}$ \\
\hline \hline
\end{tabular}

TABLE III

STEP RESPONSE CONFIGURATIONS $\left(x_{(2)}\right.$ DENOTES SECOND STATE)

\begin{tabular}{ccccc}
\hline \hline $\begin{array}{c}\text { Observer } \\
\text { Realisation }\end{array}$ & $\begin{array}{c}\text { Reference } \\
\text { Value }\end{array}$ & $\begin{array}{c}\text { Disturbance } \\
\text { Magnitude }\end{array}$ & Controller & Constraints \\
\hline $1(\mathrm{c})$ & 0.01 & 0.003 & Baseline & - \\
$1(\mathrm{c})$ & 0.01 & 0.003 & MPC & $|u| \leq 20,\left|x_{(2)}\right| \leq 0.04$ \\
$1(\mathrm{c})$ & 0.1 & 0.03 & Baseline & - \\
$1(\mathrm{c})$ & 0.1 & 0.03 & MPC & $|u| \leq 20,\left|x_{(2)}\right| \leq 0.04$ \\
\hline \hline
\end{tabular}

$\left[A K_{f 1}^{T}, A K_{f 2}^{T}\right]=[0.2210,0.1174,7.7703]$. and cost matrices $Q=$ $\left[\begin{array}{ll}0.0212 & 0.0019 \\ 0.0019 & 2.3454\end{array}\right] \times 10^{3}, R=0.0017 S=\left[\begin{array}{c}0.4617 \\ -0.2227\end{array}\right] \times 10^{-4}$, $P=\left[\begin{array}{ll}0.9346 & 0.3946 \\ 0.3946 & 5.4712\end{array}\right]$. Figure 1 shows the responses to a step reference change at time $t=0$ and a step input disturbance at time $t=100$, for the magnitudes and controllers shown in Table III. Process and measurement noise are omitted for clarity. The MPC controller performs identically to the baseline controller for the smaller reference and disturbance steps. For the larger reference and disturbance steps, the input and state constraints (which would be violated by the baseline controller), are respected and the first state converges to the reference.

\section{CONCLUSIONS}

An approach to designing a constrained output-feedback predictive controller with the objective of reproducing the same small-signal properties as a pre-existing output-feedback linear time invariant controller has been proposed, with systematic guidelines to select the appropriate (non-unique) realisation of the resulting state observer. A class of offset-free reference tracking controllers is transformed into the combination of an observer, reference pre-filter, steady-state target calculator and predictive controller. The process is demonstrated in simulation for a simple offset-free tracking controller.

\section{APPENDIX: PROOF OF SELECTED THEOREMS}

Proof of Theorem 1: Consider a (non-minimal) realisation of the baseline controller

$$
K_{0}(z)=\left[\begin{array}{cc|c}
A_{K} & 0 & B_{K} \\
A_{E K} & A_{E} & B_{E} \\
\hline C_{K} & 0 & D_{K}
\end{array}\right]
$$

where $A_{E K} \in \mathbb{R}^{\left(n_{x}-n_{K}\right) \times n_{K}}, A_{E} \in \mathbb{R}^{\left(n_{x}-n_{K}\right) \times\left(n_{x}-n_{K}\right)}, B_{E} \in$ $\mathbb{R}^{\left(n_{x}-n_{K}\right) \times n_{y}}$ have arbitrary values, and $A_{E}$ is Schur stable. $T$ is of full row rank, $\left[\begin{array}{cc}T^{T} & T^{\perp}\end{array}\right]^{T}$ is invertible, with inverse $\left[\begin{array}{ll}T^{+} & T^{\perp}\end{array}\right]$. Defining $A_{c}=A+B K_{c}$, consider the observer-based controller

$$
K_{\mathrm{obs}}(z)=\left[\begin{array}{c|c}
A_{c}\left(I-K_{f} C\right)-B D_{Q} C & A_{c} K_{f}+B D_{Q} \\
\hline K_{c}\left(I-K_{f} C\right)-D_{Q} C & K_{c} K_{f}+D_{Q}
\end{array}\right]
$$

and a change of co-ordinates $\bar{K}_{\text {obs }}(z)=\left[\begin{array}{c|c}A_{\text {obs }} & B_{\text {obs }} \\ \hline C_{\text {obs }} & D_{\text {obs }}\end{array}\right]$, where $A_{\mathrm{obs}}=\left[\begin{array}{cc}T M_{1} T^{+} & T M_{1} T^{\perp} \\ T^{\perp^{T}} M_{1} T^{+} & T^{\perp^{T}} M_{1} T^{\perp}\end{array}\right], M_{1}=A-A K_{f} C+B K_{c}-$ $B K_{c} K_{f} C-B D_{Q} C, B_{\mathrm{obs}}=\left[\begin{array}{c}T A K_{f}+T B D_{\mathrm{obs}} \\ T^{\perp^{T}} A K_{f}+T^{\perp^{T}} B D_{\mathrm{obs}}\end{array}\right], C_{\mathrm{obs}}=$ $\left[\left(K_{c}-D_{\mathrm{obs}} C\right) T^{+},\left(K_{c}-D_{\mathrm{obs}} C\right) T^{\perp}\right], D_{\mathrm{obs}}=K_{c} K_{f}+D_{Q}$. Let $K_{c}=D_{K} C+C_{K} T, A K_{f}=T^{+} B_{K}-B D_{K}+T^{\perp} K_{N}$, and $D_{Q}=$ $D_{K}-K_{c} K_{f}$, then $T M_{1}=T A+T B D_{K} C-B_{K} C+T B C_{K} T$, $B_{\mathrm{obs}}=\left[\begin{array}{ll}B_{K}^{T} & K_{N}^{T}\end{array}\right]^{T}$, and $C_{\mathrm{obs}}=\left[\begin{array}{ll}C_{K} & 0\end{array}\right]$. If (2) holds then $T M_{1}=A_{K} T$ and $T M_{1} T^{+}=A_{K}$, and $T M_{1} T^{\perp}=0 . \bar{K}_{\text {obs }}$ is related to (19) by similarity transformation, and $\bar{K}_{\text {obs }}$ is equal to (18) with $A_{E K}=T^{\perp^{T}}\left(A+B D_{K} C-T^{\perp} K_{N} C\right) T^{+}+T^{\perp^{T}} B C_{K}$, $A_{E}=T^{\perp^{T}}\left(A+B D_{K} C-T^{\perp} K_{N} C\right) T^{\perp}$, and $B_{E}=K_{N}$, which in turn is a (non-minimal) realisation of the original regulator. Therefore (19) is a (non-minimal) realisation of that too. 
Proof of Corollary 1: $K_{c} K_{f}=\left(D_{K} C+C_{K} T\right) A^{-1}\left(T^{+} B_{K}+\right.$ $\left.T^{\perp} K_{N}-B D_{K}\right)=C_{K} A_{K}^{-1}\left(B_{K} C+A_{K} T\right) A^{-1}\left(\left(T^{+}-\right.\right.$ $\left.\left.B C_{K} A_{K}^{-1}\right) B_{K}+T^{\perp} K_{N}\right)$. By rearrangement and factorisation, $T A=\left(I-T B C_{K} A_{K}^{-1}\right)\left(B_{K} C+A_{K} T\right)$. Therefore, $B_{K} C+$ $A_{K} T=\left(I-T B C_{K} A_{K}^{-1}\right)^{-1} T A$, and $K_{c} K_{f}=C_{K} A_{K}^{-1}(I-$ $\left.T B C_{K} A_{K}^{-1}\right)^{-1}\left(I-T B C_{K} A_{K}^{-1}\right) B_{K}=D_{K}$.

Proof that $\left(K_{c}\left(I-K_{f} C\right)-D_{Q} C\right) T^{\perp}=0:\left(K_{c}\left(I-K_{f} C\right)-\right.$ $\left.D_{Q} C\right) T^{\perp}=\left(C_{K} T+D_{K} C-K_{c} K_{f} C-\left(D_{K}-K_{c} K_{f}\right) C\right) T^{\perp}=$ $C_{K} T T^{\perp}=0$.

\section{ACKNOWLEDGMENTS}

The authors would like to acknowledge the constructive comments provided by the anonymous reviewers and the associate editor.

\section{REFERENCES}

[1] J. M. Maciejowski, Predictive Control with Constraints. Harlow, U.K.: Prentice Hall/Pearson Education, 2002.

[2] E. F. Camacho and C. Bordons, Model predictive control, ser. Advanced Textbooks in Control and Signal Processing. London: Springer, 2004.

[3] J. B. Rawlings and D. Q. Mayne, Model predictive control: Theory and design. Nob Hill Publishing, 2009.

[4] S. J. Qin and T. A. Badgwell, "A survey of industrial model predictive control technology," Contr. Eng. Practice, vol. 11, no. 7, pp. 733-764, Jul. 2003.

[5] R. Kalman, "When is a linear control system optimal?" Trans. ASME, J. Basic Engineering, Series D, vol. 86, pp. 81-90, 1964.

[6] S. Boyd, L. E. Ghaoui, E. Feron, and V. Balakrishnan, Linear Matrix Inequalities in Systems and Control Theory. Philadelphia, PA: SIAM, 1994.

[7] S. Di Cairano and A. Bemporad, "Model predictive control tuning by controller matching," IEEE Trans Automat Contr, vol. 55, no. 1, pp. 185-190, Jan. 2010.

[8] E. Kreindler and A. Jameson, "Optimality of linear control systems," IEEE Trans Automat Contr, vol. 17, no. 3, pp. 349-351, Jun. 1972.

[9] C. Rowe and J. Maciejowski, "Tuning MPC using $H_{\infty}$ loop shaping," in Proc. American Control Conf., vol. 2, Chicago, IL, USA, Jun 28-30 2000, pp. 1332-1336.

[10] D. McFarlane and K. Glover, "A loop-shaping design procedure using $H_{\infty}$ synthesis," IEEE Trans Automat Contr, vol. 37, no. 6, pp. 759-769, Jun. 1992.

[11] D. J. Bender and R. A. Fowell, "Computing the estimator-controller form of a compensator," International Journal of Control, vol. 41, no. 6, pp. 1565-1575, 1985.

[12] D. J. Bender, "Some considerations for estimator-based compensator design," International Journal of Control, vol. 41, no. 6, pp. 1577-1588, Jun. 1985

[13] D. Alazard and P. Apkarian, "Exact observer-based structures for arbitrary compensators," Int J Robust Nonlinear Control, vol. 9, no. 2, pp. 101-118, Feb. 1999.

[14] F. Delmond, D. Alazard, and C. Cumer, "Cross standard form: a solution to improve a given controller with $\mathrm{H} 2$ or H-infinity specifications," International Journal of Control, vol. 79, no. 4, pp. 279-287, Apr. 2006.

[15] D. Alazard, "Cross standard form for generalized inverse problem: application to lateral flight control of a highly flexible aircraft," in Proc. Int. Conf. Nonlinear Problems in Aviation and Aerospace, Melbourne (Florida), May 2002.

[16] O. Voinot, D. Alazard, P. Apkarian, S. Mauffrey, and B. Clement, "Launcher attitude control: Discrete-time robust design and gainscheduling," Control Engineering Practice, vol. 11, no. 11, pp. 1243 1252, Nov. 2003.

[17] J. M. Maciejowski, "Reverse engineering existing controllers for MPC design," in IFAC Symp. System Structure and Control, Iguassu Falls, Brazil, Oct 17-19 2007.

[18] E. N. Hartley and J. M. Maciejowski, "Initial tuning of predictive controllers by reverse engineering," in Proc. European Control Conf., Aug 23-26 2009, pp. 725-730.

[19] D. A. Joosten and J. M. Maciejowski, "MPC design for fault-tolerant flight control purposes based upon an existing output feedback controller," in Proc. IFAC Symp. Fault Detection, Supervision and Safety of Technical Processes, Barcelona, Spain, Jun 30-Jul 3 2009, pp. 253-258.
[20] E. N. Hartley and J. M. Maciejowski, "Designing MPC controllers by reverse-engineering existing LTI controller," University of Cambridge Department of Engineering, Tech. Rep. CUED/F-INFENG/TR.671, Sep 2011.

[21] K. R. Muske and T. A. Badgwell, "Disturbance modeling for offset-free linear model predictive control," J. Process Contr., vol. 12, no. 5, pp. 617-632, 2002.

[22] G. Pannocchia and J. B. Rawlings, "Disturbance models for offset-free model predictive control," AIChE J., vol. 49, no. 2, pp. 426-437, Feb 2003.

[23] U. Maeder, F. Borrelli, and M. Morari, "Linear offset-free model predictive control," Automatica, vol. 45, no. 10, pp. 2214-2222, Oct. 2009.

[24] O. Voinot, D. Alazard, and B. Clement, "Unstationary control of a launcher using observer-based structures," in Proc. American Control Conf., vol. 4. Denver, CO: IEEE, Jun 04-06 2003, pp. 3466-3471.

[25] V. L. Syrmos, C. T. Abdallah, P. Dorato, and K. Grigoriadis, "Static output feedback-A survey," Automatica, vol. 33, no. 2, pp. 125-137, Feb 1997.

[26] P. V. Pakshin and A. V. Ryabov, "A static output feedback control for linear systems," Automation and Remote Control, vol. 65, no. 4, pp. 559-566, Apr. 2004.

[27] D. Rosinová and V. Veselé, "Robust static output feedback for discretetime systems - LMI approach," Periodica Polytechnica, vol. 48, no. 3-4, pp. 151-163, 2004.

[28] K. H. Lee, "Suboptimal digital LQ output feedback control design via LMI relaxations," Optim Control Appl Meth, vol. 28, no. 6, pp. 451-465, Nov. 2007.

[29] D. Q. Mayne, J. B. Rawlings, C. V. Rao, and P. O. M. Scokaert, "Constrained model predictive control: Stability and optimality," Automatica, vol. 36, no. 6, pp. 789-814, Jun 2000.

[30] E. C. Kerrigan and J. M. Maciejowski, "Feedback min-max model predictive control using a single linear program: robust stability and the explicit solution," Int. J. Robust Nonlinear Control, vol. 14, pp. 395-413, 2004.

[31] L. Vandenberghe and S. Boyd, "Semidefinite programming," SIAM Rev., vol. 38, no. 1, pp. 49-95, Mar. 1996.

[32] M. J. Sidi, Spacecraft dynamics and control: A practical engineering approach. Cambridge University Press, 1997.

[33] D. Peaucelle, D. Henrion, Y. Labit, and K. Taitz, User's Guide for SeDuMi Interface, 2002.

[34] J. Löfberg, "YALMIP: A toolbox for modeling and optimization in MATLAB," in Proc. CACSD Conf., Taipei, Taiwan, R.O.C., 2004. 